\title{
Effect of Arrangement of Ag Nanodomes on Performance of Plasmonic Sensor
}

\author{
Mana Toma, Yuji Itakura, and Kotaro Kajikawa \\ Department of Electrical and Electronic Engineering, School of Engineering, Tokyo Institute of Technology, \\ Nagatsuta-cho 4259 G2-8, Midori-ku, Yokohama, Kanagawa 226-8503, Japan
}

(Received September 18, 2020; accepted December 4, 2020)

Keywords: plasmon, refractive index sensor, biosensor, metal nanostructure arrays

In this study, we investigated the effect of the arrangement of Ag nanodomes on the performance of a plasmonic refractive index sensor. As a template of Ag nanodomes, polystyrene (PS) bead layers with surface coverages of 56 and $90 \%$ and a multilayered structure were created. The optical properties and sensing performance of Ag nanodomes were characterized by reflection spectroscopy and bulk refractive index sensing. The Ag nanodomes created on the closely packed and multilayered PS beads exhibited similar reflection spectra and bulk refractive index sensitivity. The differences between these Ag nanodomes were in the resonance wavelength and the corresponding reflective colors. By reducing the surface coverage of PS beads, the reflectance decreased, the resonance dip appeared at a longer wavelength, and the bulk refractive index sensitivity was improved to $516 \mathrm{~nm}$ per refractive index unit (RIU). The experimental results revealed that the arrangement of Ag nanodomes has a notable influence on their optical properties and needs to be optimized to improve the performance of plasmonic biosensors.

\section{Introduction}

Plasmonic biosensors have been developed as a powerful method for analyzing biomolecular interactions and detecting a variety of biomolecules with high sensitivity. ${ }^{(1-3)}$ In plasmonic biosensors based on refractometric detection, changes in the local refractive index arising from the binding of target molecules on the sensor surface can be detected via optical signals such as reflectance and transmittance signals. The key advantages of plasmonic biosensors are their ability for the real-time kinetic measurement of biomolecular interactions, parallel detections of multiple target analytes, and flexibility of optical setups. Nowadays, the development of portable and easy-to-use plasmonic biosensors is an active issue due to the increasing demand in various fields such as home medical care, preventive care, food safety, and quarantine. ${ }^{(4,5)}$ The recent advances in plasmonic materials consisting of metal nanostructure arrays have provided a platform to establish portable sensing devices, because the resonant condition of surface plasmons and the sensitivity are highly tunable via the shape and arrangement of the metal

*Corresponding author: e-mail: toma.m.aa@m.titech.ac.jp

https://doi.org/10.18494/SAM.2021.3071 
nanostructures. ${ }^{(6-9)}$ Plasmonic coloration is one of the attractive optical properties obtained with metal nanostructure arrays, because RGB values in a photograph can be directly used as sensor signals. ${ }^{(9-13)}$ Nevertheless, it is still challenging to realize a reliable colorimetric plasmonic biosensor with high sensitivity.

In a previous study, we proposed the use of metal nanodome arrays for plasmonic biosensors based on colorimetric detection, and demonstrated the quantitative detection of biomolecules by measuring shifts of the Hue angle in HSV color space. ${ }^{(14)}$ Metal nanodome arrays are metalcoated polystyrene (PS) bead layers as illustrated in Fig. 1, which are created by a simple bottom-up process. This structure exhibits reflective colors, which can be controlled via the dome diameter (defined as the PS bead diameter $\phi$ ), the metal species, and the metal thickness. ${ }^{(15)}$ It was previously revealed that the refractive index sensitivity is improved with increasing diameter of the metal nanodomes. On the other hand, a suitable dome diameter for colorimetric detection is around $200 \mathrm{~nm}$, at which the shift of the resonance wavelength in reflection spectra is visible as a change in color of the sensor surface. Since the formation of PS bead layers relies on a self-assembly process, the arrangement of the PS beads is partially inhomogeneous. The inhomogeneous arrangement of the metal nanodomes may result in changes in optical properties including the performance of plasmonic biosensors.

Herein, we report an experimental investigation of the effect of the arrangement of $\mathrm{Ag}$ nanodomes on the performance of plasmonic sensors. In this study, the diameter of PS beads used as a template was fixed to $200 \mathrm{~nm}$, and the arrangement of Ag nanodomes was controlled via the concentration of the PS bead solution used for spin-coating. The changes in the optical properties of $\mathrm{Ag}$ nanodomes were characterized by reflection spectroscopy. Then, the fundamental performance of plasmonic sensors was evaluated as the bulk refractive index sensitivity.

\section{Materials and Methods}

\subsection{Materials}

The PS bead solution (carboxylate-coated, 2.6\% w/v, $200 \mathrm{~nm} \phi$ ) was purchased from Polysciences. Triton X was obtained from Sigma Aldrich. Ethylene glycol (EG) was purchased from Wako Pure Chemical Corporation.

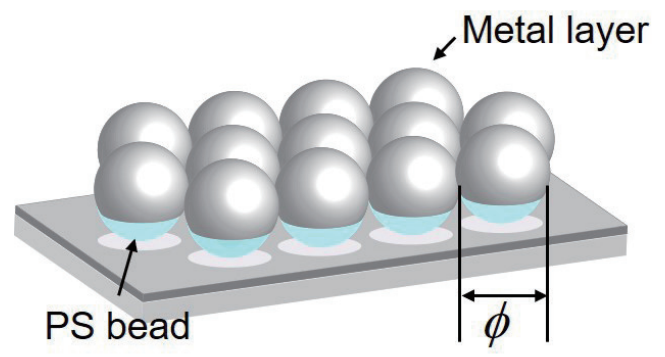

Fig. 1. (Color online) Schematic image of Ag nanodomes. 


\subsection{Fabrication of Ag nanodomes}

Ag nanodomes of $200 \mathrm{~nm}$ diameter were fabricated by a similar procedure as described in a previous paper. ${ }^{(14)}$ Briefly, an aqueous solution of PS beads was mixed with methanol with a volume ratio of $1: 3$, and a spike of Triton $\mathrm{X}$ was added as a surfactant $(0.2 \mathrm{v} \%)$. The surfactant and methanol in the spin-coating solution are important for creating the PS bead layer. The concentration of the PS bead solution was adjusted from 0.72 to $1.72 \% \mathrm{w} / \mathrm{v}$ to control the surface coverage. The PS bead solution with a volume of $30 \mu \mathrm{L}$ was spin-coated onto a glass substrate cut to $2.6 \times 2.6 \mathrm{~cm}^{2}$ with a typical spin speed of $1000 \mathrm{rpm}$ for $6 \mathrm{~s}$. The solvent was slowly dried by covering the substrate with a glass Petri dish. An Ag layer of $50 \mathrm{~nm}$ thickness was deposited by thermal vapor deposition with a thin $\mathrm{Cr}$ layer used as an adhesion layer. The thickness of the Ag layer was measured using a quartz microbalance. In addition, a thin Au layer of $5 \mathrm{~nm}$ thickness was deposited using a thermal evaporator to protect the Ag film from oxidation in an aqueous solution. Note that the influence of the Au layer thickness on the optical properties of $\mathrm{Ag}$ nanodomes is not significant at this thickness. We refer to the resulting structure as $\mathrm{Ag}$ nanodomes and to the substrate covered with $\mathrm{Ag}$ nanodomes as a sensor chip for simplicity. Next, the sensor chip was annealed on a hot plate at $120{ }^{\circ} \mathrm{C}$ for about $2 \mathrm{~min}$ to improve the adhesion of PS beads to the glass substrate. The arrangement of Ag nanodomes was observed by scanning electron microscopy (SEM). The surface coverage and lattice constant of Ag nanodome arrays were estimated from SEM images by using ImageJ (US National Institutes of Health, http://imagej.nih. gov/ij/).

\subsection{Experimental setup}

The laboratory-built optical setup depicted in Fig. 2 was used to take reflection spectra and images at the sensor surface. White light from a tungsten halogen lamp (HL-2000-HP, Ocean Optics) was coupled into an optical fiber (M92L01, $200 \mu \mathrm{m}, 0.22 \mathrm{NA}$, Thorlabs) and collimated by an achromatic lens (AC254-40-A-ML, Thorlabs). A color filter (FGT165M, Thorlabs) was used to increase the color temperature of the light source. The reflected light from a beam splitter (CCM1-BS013/M, Thorlabs) was irradiated to the sensor surface at normal incidence. An achromatic lens was installed between the first beam splitter and the sensor chip to reduce the spot size to approximately $1 \mathrm{~mm}$ diameter on the sensor surface. Images of the sensor chips were taken with the collimated incident light without the achromatic lens. The reflected light from the sensor surface was split into two directions by the second beam splitter. One of the reflected beams was coupled into an optical fiber connected to a spectrometer (USB4000, Ocean Optics). The other was used for imaging by a color CCD camera (acA2440-35ucMED, Basler) with a $\mathrm{C}$ mount lens (C23-5026-2M, Basler). As a reference spectrum, the reflection spectrum from an Al mirror (TFA-20C03-10, Sigma-Koki) was measured. LabVIEW software was used to obtain and analyze reflectance spectra. 


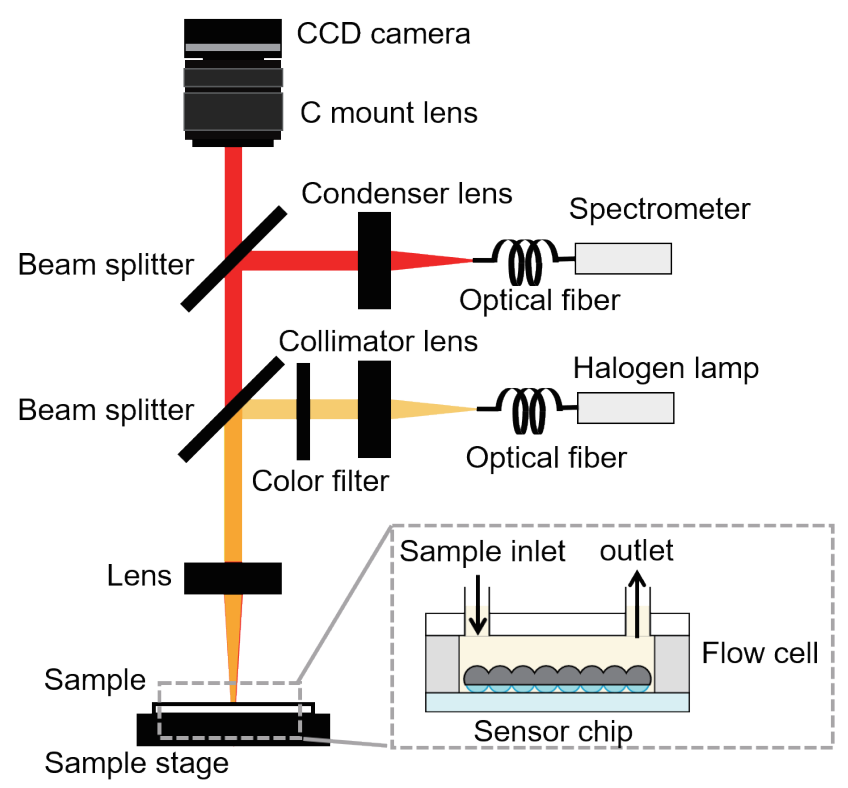

Fig. 2. (Color online) Sketch of the optical setup.

\subsection{Bulk refractive index measurements}

For bulk refractive index measurements, a flow cell of a polydimethylsiloxane (PDMS) gasket was attached on the sensor surface, as illustrated in Fig. 2, and a transparent sample solution was introduced using a syringe. The sample solutions were the mixtures of MilliQ water and EG with different volume ratios $(0,5,10,15$, and $20 \mathrm{vol} \%)$, and were sequentially injected into the flow cell. The resonance wavelength was evaluated by fitting the reflection spectra to a 10th-degree polynomial function. The wavelength range used for fitting was roughly set to within $50 \mathrm{~nm}$ of the dip wavelength. Thirty spectra were averaged to reduce the deviation of sensor signals. The refractive indexes of water and EG were obtained from the literature. ${ }^{(16)}$ The refractive index of the sample solution was approximated as the mean refractive index of the mixture at the volume ratio.

\section{Results and Discussion}

\subsection{Characterization of Ag nanodomes}

First, the surface coverage and arrangement of the Ag nanodomes were investigated by SEM. Representative SEM images are shown in Fig. 3, and the characteristics of Ag nanodomes in terms of surface coverage, lattice constant, and arrangement are summarized in Table 1. As shown in Fig. 3(b), the Ag nanodomes formed two-dimensionally and hexagonally packed multiple crystals when the surface coverage was $90 \%$. When the concentration of the PS bead solution for spin-coating was lower, the size of the PS bead assembly decreased and connected clusters were observed as shown in Fig. 3(a). With increasing concentration of the spin-coating 


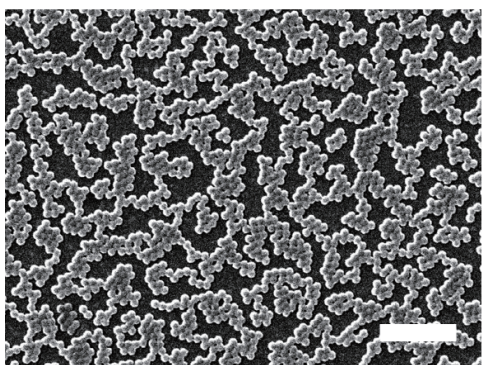

(a)

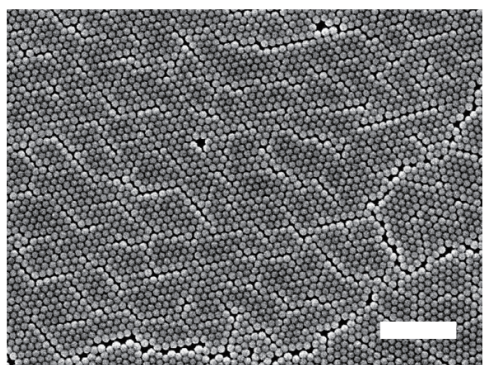

(b)

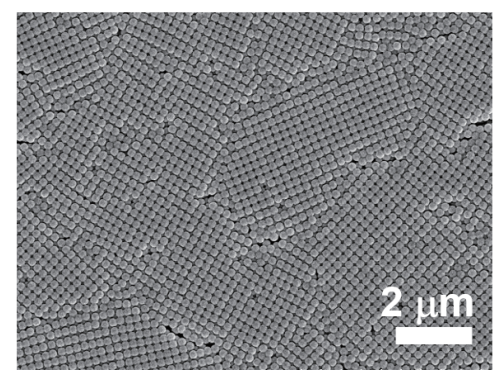

(c)

Fig. 3. SEM images of Ag nanodome arrays created on PS bead layer with (a) low surface coverage, (b) high surface coverage, and (c) multiple layers. The scale bars indicate $2 \mu \mathrm{m}$.

Table 1

Summary of concentration of PS bead solution, surface coverage, lattice constant, and arrangement of Ag nanodome arrays.

\begin{tabular}{lcccc}
\hline Surface coverage & Concentration (\% w/v) & Surface coverage (\%) & Lattice constant (nm) & Arrangement \\
\hline Low & 0.72 & 56 & - & Cluster \\
High & 1.44 & 90 & 180 & Hexagonal \\
Multilayered & 1.72 & - & 200 & Square \\
\hline
\end{tabular}

solution, the PS beads formed multiple layers, and a square-array structure was observed in the top layer as shown in Fig. 3(c). Under these experimental conditions, the number of PS bead layers was two or three. The lattice constants of the Ag nanodomes were obtained by fast Fourier transform analysis of the SEM images using ImageJ. The lattice constant of the hexagonally packed structure was $180 \mathrm{~nm}$, which is slightly smaller than that expected from the PS bead size. The lattice constant of the square lattice was $200 \mathrm{~nm}$.

Figures 4(a)-4(c) show a series of reflection spectra and photographs of Ag nanodomes taken at normal incidence. It was clearly observed that the optical properties of $\mathrm{Ag}$ nanodomes significantly changed with their arrangement. When the surface coverage of the Ag nanodomes was low and the structures formed clusters, the reflectance was significantly suppressed and a resonant dip appeared at $526 \mathrm{~nm}$ in air, as shown in Fig. 4(a). The reflective color changed from dark purple to dark blue when water was introduced. Strong light scattering was observed from these Ag nanodomes. Therefore, although the reflective color of this structure was dark purple, the color recognized by visual observation was a rather pale purple. In the case of the Ag nanodome hexagonal arrays, there was no clear resonance dip in the visible wavelength region, as shown in Fig. 4(b), when the sensor surface was in contact with air. Owing to the high reflectance over the visible wavelength region, the reflective color of this structure was whitish gold. When water was introduced onto the surface of the Ag nanodomes, a resonance dip denoted by $\lambda_{d}$ appeared at $459 \mathrm{~nm}$ and the reflective color was tinged with yellow. In the case of the square-array structure, the basic features of the resonance curve were similar to those of the hexagonal arrays. As shown in Fig. 4(c), a resonance dip appeared in the reflection spectra at $426 \mathrm{~nm}$ in air, which shifted to $524 \mathrm{~nm}$ when water was applied to the sensor surface. In this wavelength range, the color of the Ag nanodomes drastically changed from yellow to 


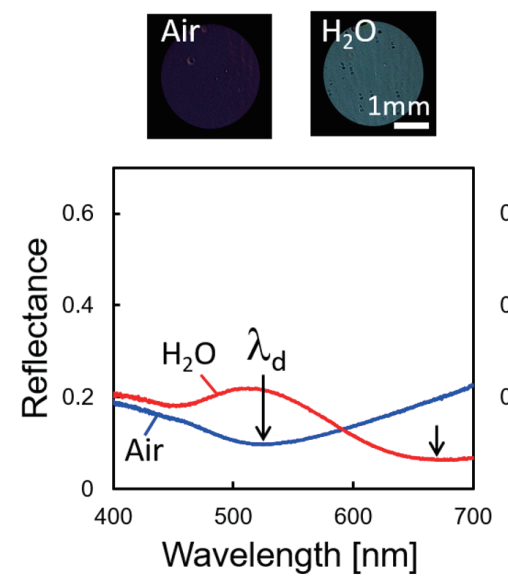

(a)
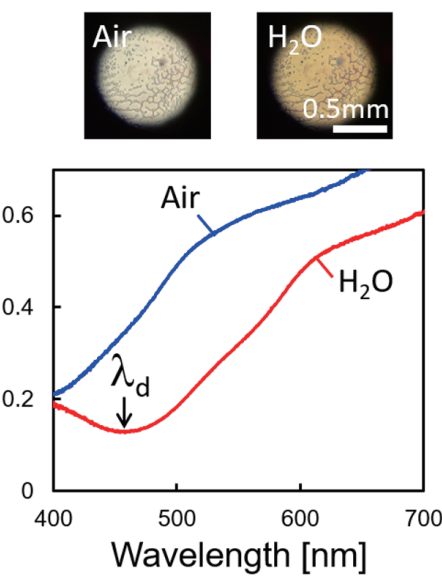

(b)
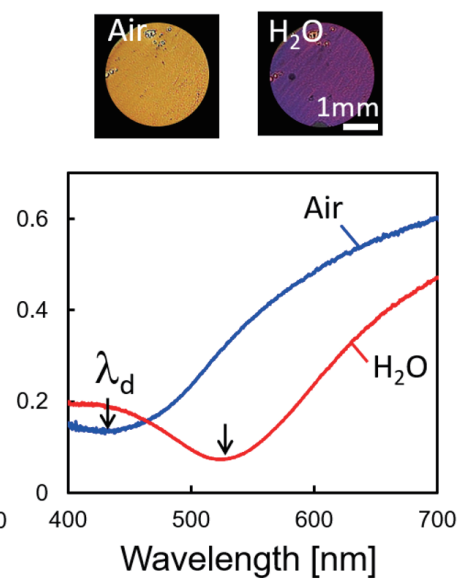

(c)

Fig. 4. (Color online) Representative photographs and reflectance spectra of Ag nanodomes arranged in (a) connected clusters, (b) hexagonal arrays, and (c) square arrays, taken in contact with air and water.

purple. According to the literature, the resonance condition of metal nanodome structures strongly depends on their arrangement. ${ }^{(17,18)}$ We consider that the redshift of the resonance wavelength with the formation of the square lattice is due to the expansion of the gap between the nanodomes. In the case of $\mathrm{Ag}$ nanodomes forming clusters, there are two possible reasons for the change in the reflection property: the different mean number of $\mathrm{Ag}$ nanodomes forming a single domain and the increase in the mean distance among the Ag nanodomes. To clarify the plasmonic properties of the Ag nanodomes, a further investigation by numerical simulation is required. The reflection spectra of closely packed Ag nanodomes created with the same PS beads were studied previously. ${ }^{(15)}$ In the study, a resonance dip appeared at around $490 \mathrm{~nm}$ in air, which is significantly longer than the wavelength observed in Fig. 4(b). A possible reason for the difference in the optical properties is the method of metal deposition. Namely, thermal evaporation and RF sputtering were used in this study and Ref. 15, respectively. We infer that slight differences in the shape or surface roughness of the metal nanodomes cause changes in the resonance condition.

\subsection{Bulk refractive index sensing}

To compare the sensing performances of $\mathrm{Ag}$ nanodomes with different arrangements, bulk refractive index measurement was performed as described in Sect. 2. Figure 5 shows a typical sensor response obtained with the Ag nanodomes fabricated on the PS bead multilayer. Initially, the sensor surface was in contact with water and the dip wavelength was recorded as a baseline. After the sensor signal became stable, a sample solution containing EG was sequentially injected into the flow cell using a syringe. As shown in Fig. 5, the dip wavelength $\lambda_{d}$ immediately underwent a redshift when the concentration of EG was increased then reached a plateau. Reversible sensor signals were obtained when the concentration of EG was decreased from 20 to $0 \mathrm{v} \%$. The shape of the reflection spectra was retained both in the water and in 


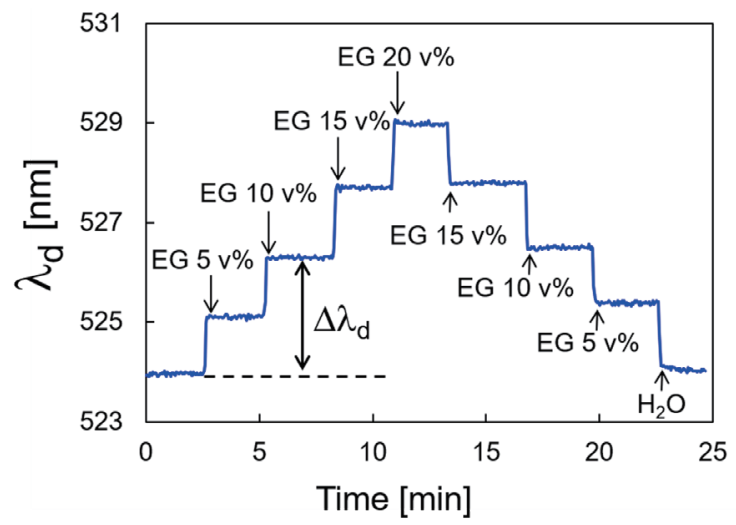

Fig. 5. (Color online) Time-dependent measurement of resonance wavelength $\lambda_{d}$ with the injection of sample solutions containing EG with various concentrations.

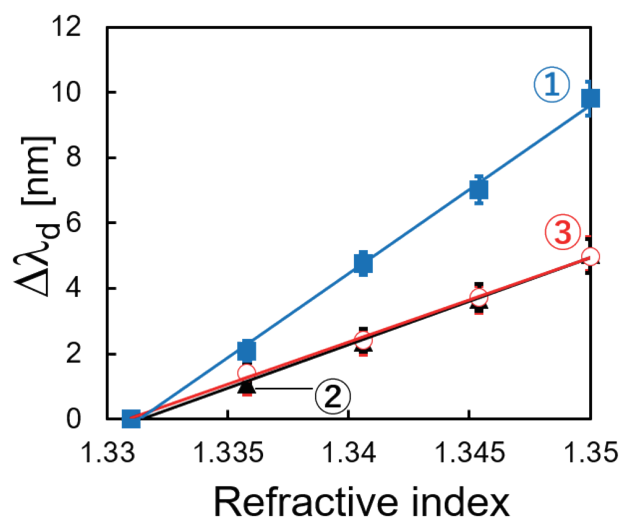

Fig. 6. (Color online) Plot of $\Delta \lambda_{d}$ as a function of refractive index of sample solution. Numbers correspond to those in Table 2.

the solution containing EG. The shift of the resonance wavelength $\Delta \lambda_{d}$ from the baseline is plotted as a function of the refractive index of the sample solution in Fig. 6. The experiments were repeated at least three times and average values were plotted. $\Delta \lambda_{d}$ and the refractive index of the sample solution show a linear correlation regardless of the arrangement of the $\mathrm{Ag}$ nanodomes. Here, we evaluate the bulk refractive index sensitivity $\left(S_{R I}\right)$ and the resolution of the refractive index $\left(\sigma_{R I}\right)$ as standard parameters to evaluate the actual sensing performance of Ag nanodomes. ${ }^{(1)} S_{R I}$ was determined from the slope of the linear fitted function, and $\sigma_{R I}$ was determined by substituting the standard deviation of the sensor signals $\left(\sigma_{n}\right)$ into the fitted function as $\sigma_{n}=S_{R I} / \sigma_{R I}{ }^{(1)}$ The values of $S_{R I}, \sigma_{R I}$, and $\sigma_{n}$ are summarized in Table 2 . The Ag nanodomes with high surface coverage and multilayered structures exhibited similar values of $S_{R I}$ and $\sigma_{R I}$ of 278 and $258 \mathrm{~nm}$ per refractive index unit (RIU), and $2.0 \times 10^{-4}$ and $1.0 \times$ $10^{-4}$ RIU, respectively. $S_{R I}$ was significantly improved to $516 \mathrm{~nm} / \mathrm{RIU}$ by reducing the surface coverage of $\mathrm{Ag}$ nanodomes, while $\sigma_{R I}$ was slightly higher than that of the highly dense or multilayered structure due to the higher value of $\sigma_{n}$. Since the sensitivity of plasmonic sensors is determined by the strength and the distribution of the plasmonic field where the changes in the refractive index occur, ${ }^{(19)}$ we infer that the Ag nanodomes that formed clusters have an expanded or stronger plasmonic field than that of densely packed Ag nanodomes. According to the literature, $S_{R I}$ can reach about $1000 \mathrm{~nm} / \mathrm{RIU}$ for localized surface plasmon resonance (LSPR) sensors. ${ }^{(20,21)}$ The value of $\sigma_{R I}$ obtained in this study is equal to that of an LSPR sensor, even though the value of $S_{R I}$ is inferior. We expect that a higher $\sigma_{R I}$ would be obtained by increasing the signal-to-noise ratio by improving the optical setup and further optimizing the arrangement of Ag nanodomes.

In the previous section, we reported that the Ag nanodomes created in this study have different optical properties from those in the previous study, which may be due to the difference in the fabrication method. Therefore, a difference in $S_{R I}$ was also expected. Considering this point, we concluded that there was no notable difference between the Ag nanodomes fabricated in this study and those in the previous study. ${ }^{(15)}$ 
Table 2

Summary of bulk refractive sensitivity $S_{R I}$, resolution $\sigma_{R I}$, and $\sigma_{n}$.

\begin{tabular}{lcccc}
\hline & Arrangement & $S_{R I}(\mathrm{~nm} / \mathrm{RIU})$ & $\sigma_{R I}(\mathrm{RIU})$ & $\sigma_{n}(\mathrm{~nm})$ \\
\hline$(1)$ & Cluster & 516 & $4 \times 10^{-4}$ & 0.21 \\
$(2)$ & Hexagonal & 278 & $2 \times 10^{-4}$ & 0.056 \\
$(3)$ & Square & 258 & $1 \times 10^{-4}$ & 0.026 \\
\hline
\end{tabular}

\section{Conclusion}

We studied the influence of the arrangement of Ag nanodomes on their optical properties and the performance of a plasmonic sensor by reflection spectroscopy. Three types of Ag nanodome structures with different arrangements were created using a spin-coated PS bead layer: connected clusters, hexagonally packed arrays, and square arrays. The resonance wavelength and the corresponding reflective colors drastically changed with the arrangement of $\mathrm{Ag}$ nanodomes. The Ag nanodomes arranged in hexagonally packed and square arrays exhibited similar reflection properties and sensing performance, although the Ag nanodomes arranged in square arrays exhibited a resonance dip at a longer wavelength. With decreasing surface coverage of the PS beads, the resonance dip appeared at a longer wavelength, and the bulk refractive index sensitivity was improved from $278 \mathrm{~nm} / \mathrm{RIU}$ obtained with the hexagonally packed structure to $516 \mathrm{~nm} / \mathrm{RIU}$. For Ag nanodomes with a fixed diameter, a higher sensing performance in terms of refractive index sensitivity is obtained with clusters rather than closely packed structures.

\section{Acknowledgments}

This work was partially supported by JSPS KAKENHI Grant Number JP 20K14748 (a Grant-in-Aid for Early-Career Scientists) and Izumi Science and Technology Foundation. The authors thank the Materials Analysis Division, Open Facility, Tokyo Institute of Technology, for help in the SEM analysis.

\section{References}

1 J. Homola: Chem. Rev. 108 (2008) 462. https://doi.org/10.1021/cr068107d

2 J. N. Anker, W. P. Hall, O. Lyandres, N. C. Shah, J. Zhao, and R. P. Van Duyne: Nat. Mater. 7 (2008) 442. https://doi.org/10.1038/nmat2162

3 M. Bauch, K. Toma, M. Toma, Q. Zhang, and J. Dostalek: Plasmonics 9 (2013) 781. https://doi.org/10.1007/ s11468-013-9660-5

4 O. Tokel, F. Inci, and U. Demirci: Chem. Rev. 114 (2014) 5728. https://doi.org/10.1021/cr4000623

5 C. Lertvachirapaiboon, A. Baba, K. Shinbo, and K. Kato: Anal. Methods 10 (2018) 4732. https://doi. org/10.1039/c8ay01561a

6 A. E. Cetin, A. F. Coskun, B. C. Galarreta, M. Huang, D. Herman, A. Ozcan, and H. Altug: Light Sci. Appl. 3 (2014) e122. https://doi.org/10.1038/1sa.2014.3

7 A. Belushkin, F. Yesilkoy, J. J. González-López, J. C. Ruiz-Rodríguez, R. Ferrer, A. Fàbrega, and H. Altug: Small 16 (2020) 1906108. https://doi.org/10.1002/smll.201906108

8 D. Kawasaki, H. Yamada, K. Maeno, K. Sueyoshi, H. Hisamoto, and T. Endo: ACS Appl. Nano Mater. 2 (2019) 4983. https://doi.org/10.1021/acsanm.9b00930 
9 X. H. Wang, T. W. Chang, G. H. Lin, M. R. Gartia, and G. L. Liu: Anal.1 Chem. 89 (2017) 611. https://doi. org/10.1021/acs.analchem.6b02484

10 S. Kim, Y. Lee, J. Y. Kim, J. H. Yang, H. J. Kwon, J. Y. Hwang, C. Moon, and J. E. Jang: Biosens. Bioelectron. 126 (2019) 743. https://doi.org/10.1016/j.bios.2018.11.048

11 M. R. Gartia, A. Hsiao, A. Pokhriyal, S. Seo, G. Kulsharova, B. T. Cunningham, T. C. Bond, and G. L. Liu: Adv. Opt. Mater. 1 (2013) 68. https://doi.org/10.1002/adom.201200040

12 S. Shinohara, D. Tanaka, K. Okamoto, and K. Tamada: Phys. Chem. Chem. Phys. 17 (2015) 18606. https://doi. org/10.1039/c5cp02564h

13 N. S. King, L. Liu, X. Yang, B. Cerjan, H. O. Everitt, P. Nordlander, N. J. Halas, and K. E. T. Al: ACS Nano 9 (2015) 10628. https://doi.org/10.1021/acsnano.5b04864

14 M. Toma and K. Tawa: Nanoscale Adv. (2019). https://doi.org/10.1039/C9NA00315K

15 M. Toma, R. Tanaka, and K. Tawa: Jpn. J. Appl. Phys. 59 (2020) SDDF03. https://doi.org/10.7567/1347-4065/ ab4ea7

16 H. El-Kashef: Physica B 279 (2000) 295. https://doi.org/10.1016/s0921-4526(99)00856-X

17 A. I. Maaroof, M. B. Cortie, N. Harris, and L. Wieczorek: Small 4 (2008) 2292. https://doi.org/10.1002/ sml1.200800203

18 H.-Y. Wu, C. J. Choi, and B. T. Cunningham: Small 8 (2012) 2878. https://doi.org/10.1002/smll.201200712

19 M. Piliarik, P. Kvasnička, N. Galler, J. R. Krenn, and J. Homola: Opt. Express 19 (2011) 9213. https://doi. org/10.1364/OE.19.009213

20 Y. Shen, J. Zhou, T. Liu, Y. Tao, R. Jiang, M. Liu, G. Xiao, J. Zhu, Z.-K. Zhou, X. Wang, C. Jin, and J. Wang: Nat. Commun. 4 (2013) 2381. https://doi.org/10.1038/ncomms3381

21 M. Toma, K. Cho, J. B. Wood, and R. M. Corn: Plasmonics 9 (2013) 765. https://doi.org/10.1007/s11468-013$\underline{9657-0}$ 